\title{
Belphégor
}

\section{Tra biografia e critica letteraria. Qualche nota su Sweet Salgari di Paolo Bacilieri}

\section{Alberto Brambilla}

\section{(2) OpenEdition}

1 Journals

\section{Edizione digitale}

URL: http://journals.openedition.org/belphegor/175

DOI: $10.4000 /$ belphegor. 175

ISSN: 1499-7185

Editore

LPCM

\section{Notizia bibliografica digitale}

Alberto Brambilla, «Tra biografia e critica letteraria. Qualche nota su Sweet Salgari di Paolo Bacilieri », Belphégor [En ligne], 11-1 | 2013, mis en ligne le 29 avril 2013, consulté le 22 septembre 2020. URL : http://journals.openedition.org/belphegor/175 ; DOI : https://doi.org/10.4000/belphegor.175

Questo documento è stato generato automaticamente il 22 settembre 2020.

\section{(c) (i) (9)}

Belphégor est mis à disposition selon les termes de la Licence Creative Commons Attribution - Pas d'Utilisation Commerciale - Pas de Modification 4.0 International. 


\section{Tra biografia e critica letteraria. Qualche nota su Sweet Salgari di Paolo Bacilieri}

Alberto Brambilla

NOTIZIA

Paolo Bacilieri, Sweet Salgari, Bologna-Parigi, Coconino Press-Fandango, 2012, ISBN 978-88-7618-206-8, 17 euro, pp. 156 
1 Come è ormai da tempo assodato, i graphic novels (o, se vogliamo, i 'romanzi a fumetti) costituiscono una delle esperienze più interessanti dal punto di vista artistico e più in generale culturale di questi ultimi anni. Esse non solo mescolano abilmente diversi codici, ma reinterpretano o creano ex novo dei generi letterari che quasi sempre approdano a prodotti difficilmente riconducibili alle tipologie classiche; e in tal modo aprono a loro volta la via ad ulteriori esperienze. Un esempio notevole in questo senso è costituito dal recente libro di Paolo Bacilieri, Sweet Salgari (Coconino PressFandango, Bologna-Parigi, 2012, ISBN 978-88-7618-206-8, 17 euro, pp. 156), che apparentemente si presenta come una classica biografia per immagini di Emilio

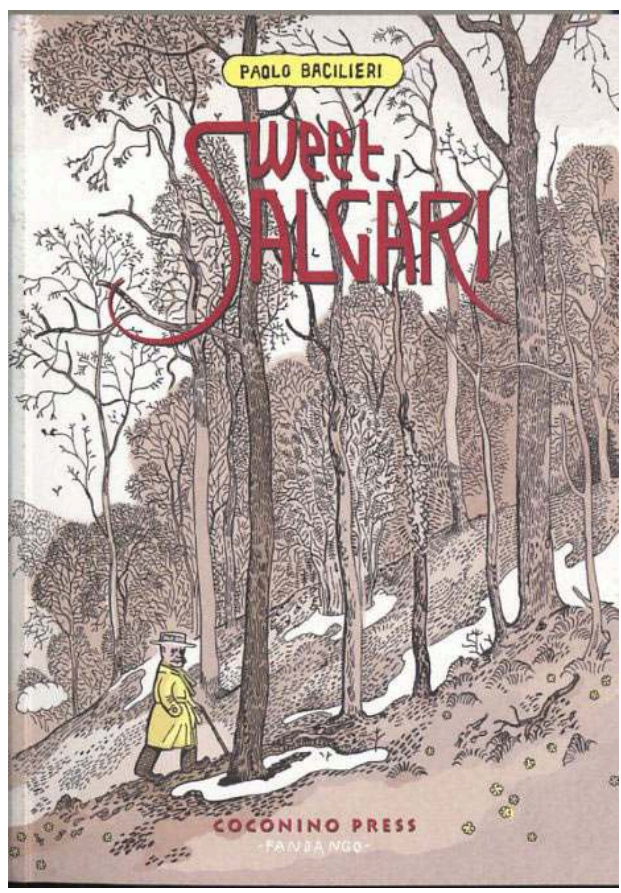
Salgari (1862-1911). Una biografia, va subito aggiunto, affettuosa e quasi familiare, in cui l'autore (per altro conterraneo di Salgari), mostra una sofferta e partecipata pietas nei riguardi del suo eroe. Ma insieme Sweet Salgari si rivela una ricostruzione oggettiva e documentata, in cui Bacilieri dimostra di aver letto a fondo i testi salgariani (in appendice, a p. 155, vi è una nota bibliografica che segnala la fonte delle citazioni) e di conoscere gli snodi principali della biografia dello scrittore veronese, nonché alcune interpretazioni critiche. Si conferma dunque in questo senso una delle caratteristiche di fondo segnalate di recente dallo storico Giovanni De Luna; il quale, dopo aver preso in considerazione alcuni particolari esempi di graphic novels, ha affermato in modo perentorio che

2 Le graphic novel sono veri libri. Le storie che raccontano sono disegnate, i loro personaggi parlano attraverso il fumetto; sono narrazioni che mettono in scena la realtà del passato e i drammi del presente partendo sempre da un robusto apparato documentario. Il loro linguaggio è totalmente affidato alle immagini, ma c'è molta storia e niente fantasy. ${ }^{1}$

Dal punto di vista della narrazione, l'attenzione di Bacilieri è puntata soprattutto sull'ultima giornata dello scrittore veronese che, come noto, non potendo più sopportare un'esistenza fatta di sacrifici e tribolazioni, si toglie la vita a Torino il 25 aprile 1911. Mescolando abilmente immagini e parole, Baccilieri descrive dunque il viaggio di Salgari, verso la terra del non ritorno. Non a caso tale drammatica esperienza è per la prima volta evocata ( $\mathrm{a}$ p. 15) dall'inquietante segnalazione di un'assenza: nello studio torinese di Salgari non c'è nessuno, ma lo sguardo del visitatore-lettore indugia sui ricordi e sugli strumenti (libri, riviste, il calamaio, una macchina da scrivere...) di un lavoro improvvisamente abbandonato sul tavolo; e poi si focalizza su un'immagine collocata nella tavola grafica in posizione centrale. È l'affermazione di un gesto simbolico ed estremo: la penna dello scrittore è stata definitivamente spezzata ed è rimasta sul manoscritto del romanzo incompiuto Testa di pietra. Finis: non scrivere significa non vivere. 
4 Da questa specie di buco nero prende come l'avvio (ma è un vortice di potenza distruttiva, a stento controllata dal rigore estetico delle immagini) la storia, che si dipana secondo alcune regole compositive ben calibrate. Il lettore salgariano intuisce da subito quale potrà essere la soluzione finale, il precipitare degli eventi, il suicidio; $\mathrm{e}$ tuttavia l'abilità di Bacilieri sta nel differire la conclusione, riempiendola di contenuti e di aspettative. In effetti attraverso la sirena grafico-acustica di un richiamo ripetuto ("Nadir!") dallo studio la scena è improvvisamente spostata all'esterno, in una dimensione che sembra ritornata normale, quotidiana. Ecco finalmente di spalle, densa ombra nera il protagonista della storia; Emilio poi appare di fronte con un viso da gattone allucinato, paglietta, bastone da passeggio e immancabile sigaretta. Lo scrittore aspetta i due figli che devono prendere il tram per andare a scuola; li accompagna alla fermata e li saluta senza particolari cerimonie. Poi passeggia come d'abitudine nel quartiere in cui da tempo vive, saluta i conoscenti, viene come al solito infastidito dai negozianti che gli chiedono di pagare i conti arretrati, e infine prende la via della collina, in mezzo a prati e a boschi, osservando i rami degli alberi che stanno gettando le prime foglie. Sembrerebbe una delle sue solite passeggiate ristoratrici, una pausa salutare dopo il solito duro esercizio allo scrittoio. E invece - noi lettori lo sappiamo bene - Emilio sta scegliendo il luogo più adatto al suicidio che ha da tempo programmato. Emilio è finalmente solo, in una sorta di doloroso nirvana che lo isola dalle mille preoccupazioni di ogni giorno (decine di pagine da riempire con nuove storie, i debiti crescenti, i figli irrequieti, la moglie ricoverata all'ospedale per problemi mentali). È disperatamente solo.

5 È evidente qui la lettura attenta delle precedenti biografie salgariane, in particolare quella di Silvino Gonzato, che appare come la fonte diretta - adeguatamente reinterpretata - di questo episodio iniziale. ${ }^{2}$ Se questa è la linea principale della narrazione, in essa si inseriscono ritmicamente dei flash back, che ricapitolano alcuni dei momenti più significativi della vita di Salgari, sia come uomo (decisivo in questo senso l'incontro con la futura moglie Ida Peruzzi, cfr. p. 74 e p. 129), che come scrittore, costituendo dunque i capitoli principali della sua biografia. La storia si apre infatti con un'ampia descrizione della Torino elegante e borghese di inizio secolo, con un riutilizzo intelligente di documenti fotografici dell'epoca, trasformati in tavole intriganti dalla mano di Bacilieri. Dall'apertura torinese (giornata del 25 aprile 1911) siamo poi trasportati a ritroso nel tempo e nello spazio, dapprima a Verona (evocata dalla rappresentazione grafica delle Arche scaligere) e poi in Valpolicella (siamo precipitati nell'anno 1872), dove si consuma la prima avventura di Emilio, a dieci anni, che cerca di arrivare al mare governando una tinozza con una pagaia rudimentale. L'impresa finirà dopo pochi metri, interrotta dall'impeto di un piccolo torrente, ma segnerà come l'inizio di una vita continuamente oscillante tra sogno e realtà, desiderio e frustrazione. Seguirà una sfortunata avventura veneziana, in cui il giovane allievo dell'Istituto Nautico, desideroso di compiere grandi imprese sul mare si imbarca come mozzo su una nave; sarà invece ingannato e derubato dal capitano dell'imbarcazione.

6 Il racconto si estenderà agli anni seguenti, toccando di nuovo Verona, dove il testardo $\mathrm{e}$ rissoso Salgari, ormai adulto, sarà coinvolto in diverse vicende (tra cui un duello con il collega giornalista Giuseppe Biasioli), e avrà occasione, in qualità di giornalista di scrivere su un'esibizione circense di un Buffalo Bill diventato ormai la patetica paradia di se stesso, sul suicidio dell'esploratore piemontese Giacomo Bove (e il tema del suicidio sarà ripreso più avanti a p. 128 con la morte di Augusto Franzoj). In seguito, 
seguendo puntualmente la biografia di Salgari, la narrazione toccherà Torino, Genova $\mathrm{e}$ approderà infine, circolarmente, ancora nel capoluogo piemontese.

7 Ma intanto, mentre come in un film si rivede a grande velocità l'intera vita di Salgari, pagina dopo pagina - a un ritmo ben più lento - continua l'ultima passeggiata di Emilio. Che trova un luogo adatto (p. 58) per il suo gesto esemplare, si spoglia con lentezza metodica, depone gli abiti, mostrandosi in tutta la sua fragilità: è ormai solo un vecchietto calvo dal volto sciupato dall'alcool e dalle lunghe veglie. Eppure ha la forza interiore (o la disperazione?) per estrarre il rasoio (pp.78-81), e incominciare ad offendere il proprio corpo, secondo una procedura che Baccilieri descrive con speciale cura, fotogramma dopo fotogramma (cfr. p. 82 e pp. 94-96, 111-112).

Come si può intuire, l'autore ha costruito una macchina complessa, in cui si intersecano contemporaneamente almeno tre linee narrative a diversa velocità ed intensità narrativa: il cammino allucinato eppure dignitosissimo di Emilio verso la morte (al ritmo di una processione rituale in cui ogni passo e ogni gesto assumono una forte connotazione simbolica); il viaggio a ritroso nel tempo, ad un ritmo diventato più rapido ed incalzante, in cui si attraversano i luoghi che hanno fatto da sfondo all'esistenza di Salgari ; infine la ricapitolazione delle esperienze più importanti della sua vita.

9 Tutto ciò non esaurisce però la ricchezza del testo proposto in Sweet Salgari, perché Bacilieri ci offre anche un'altra lettura, decisamente affascinante. Il libro incomincia con in primo piano il grande fiume Po che attraversa Torino (sullo sfondo domina infatti il profilo verticale della Mole Antonelliana), ma allinizio della pagina, in alto, scorre l'incipit di un romanzo capitale, I misteri della jungla nera ( "Il Gange, questo famoso fiume celebrato dagli indiani antichi e moderni, le cui acque sono reputate sacre da quei popoli, dopo d'aver solcato le nevose montagne dell' Himalaya..."; e in effetti la Mole specchiandosi nell'acqua sembra come disfarsi proponendo il profilo di una pagoda trasferendoci in un altro ambiente); e poi la fascia testuale prosegue invadendo le pagine successive in cui tuttavia Bacilieri ci mostra alcune tipiche 'cartoline' torinesi, contrapponendo dunque testo e ambiente. Alla fine, il feretro di Salgari sfila tra la folla silenziosa, attraversando una Torino reduce dall'Esposizione Universale, con sullo sfondo delle costruzioni simili a sontuosi palazzi di favolosi maharajah.

10 Altrove, con un procedimento analogo, Bacilieri cita dei passi di altri romanzi significativi, come Le Pantere di Algeri (p. La città del re lebbroso, Le meraviglie del 2000, per concludere con Il Corsaro Nero. Si tratta in fondo di semplici didascalie, che hanno però un valore fortemente evocativo, proponendo in continuazione il rapporto, davvero cruciale in Salgari, tra biografia e opera, scrittura e invenzione: non a caso Emilio muore invocando l'alter ego Yanez, a sua volta "l'amico della tigre" (p. 130).

11 A fare come da cerniera a queste ripartizioni interne appare insistente, a piena pagina, l'immagine dello scrittore ancorato al suo tavolo di lavoro (cfr. pp. 28, 41, 59, 83, 97, 113,119 : sovente le tavole sono affiancate dalle immagini del suicidio, quasi a proporre una specie di rapporto causa-effetto), mentre si spande, ossessiva, una sorta di colonna sonora determinata dalla pressione del pennino sulla carta (cri cri cri....). La scrittura è l'unico elemento di continuità che lega l'adolescente all'uomo diventato adulto e poi anziano. La scrittura, che nelle intenzioni di Salgari doveva incarnare il piacere dell'invenzione e dell'evasione, è invece diventata una sorta di maledizione, un destino a cui non si può sfuggire, una macchina distruttiva. 
12 In questo senso una piccola vignetta (a p. 15), assume il segno forte di un preannuncio; in essa degli operai sono impegnati nel loro lavoro sotto lo sguardo di un controllore che ha le fattezze di un Hitler. ${ }^{3} \mathrm{E}$, ugualmente, occorre ricordare l'insistenza sul rapporto di Salgari con l'editore Bemporad, descritto da Bacilieri come un cinico imprenditore, che per un verso risponde alla moglie di Salgari tranquillizzandola, per l'altro si premura di assicurarsi contro la morte del suo autore più fecondo, palesemente affetto da nevrastenia (pp. 122-124). ${ }^{4}$ Infine, mentre il cadavere di Salgari è ancora nella bara, Bacilieri ci mostra (a p. 144) i suoi parenti che discutono dei possibili introiti derivati dalla morte dello scrittore. $\mathrm{E}$ in precedenza si è colpiti dal cadavere di Salgari orrendamente mutilato, sottoposto a perizia necroscopica da parte del professor Mario Carrara di fronte a dei giovani allievi; ${ }^{5}$ parallelamente Bacilieri ci propone l'immagine non meno inquietante della moglie Ida ricoverata all'ospedale di Collegno, legata a forza al letto di contenimento. Sarà ridotta al silenzio e in qualche modo morirà con il marito (pp. 145-146).

Quanto fin qui descritto sarà sufficiente, spero, a rendere conto della complessità del lavoro di Bacilieri e a provare come sia senz'altro limitativo ridurre Sweet Salgari ad una mera biografia. Vi è perciò da aggiungere qualche altra osservazione, di ordine più generale, in quanto tale libro costituisce a suo modo anche un'operazione, forse inconsapevole, di critica letteraria. Esso infatti fornisce - sia pure nella forma narrativa tipica dei graphic novel - alcuni giudizi e interpretazioni sulla figura e l'opera di Salgari, che vanno oltre l'oggettività dei dati biografici. Basti al riguardo pensare a quanto è messo sulle labbra di Ida (alle pp. 116-117), che commenta a tavola la notizia dell'assegnazione del Premio Nobel per la letteratura (siamo nel 1907) a Rudyard Kipling (1865-1936). Ida lo contrappone polemicamente al marito ("ha la tua età, ti somiglia anche, ma è cento volte meglio di te! / Lui l'India l'ha vista veramente, ci è nato addirittura! / Lui le bestie le fa parlare, le fa vivere, per te sono solo buone da uccidere e da mangiare! / Lui è divertente, sorprendente, profondo e sincero! / Uno scrittore, un vero artista! / Tu... tu sei noioso, ripetitivo, assurdo e privo di buongusto! Non ti sai divertire e non sei divertente! / Sei solo uno scribacchino, un buffone!"), che non reagisce. Tuttavia Bacilieri fa leggere ad Ida la notizia tratta da un giornale che definisce Kipling "poeta e romanziere imperialista"; sta appunto in quell'aggettivo finale, in apparenza oggettivo un vero e proprio giudizio (per noi lettori moderni negativo) su Kipling, che è tacitamente contrapposto da Bacilieri a Salgari, scrittore certamente non imperialista, spesso dalla parte dei più deboli e dei 'ribelli'.

Ma non è questo un caso isolato. La ricostruzione biografica di Bacilieri, di solito molto precisa, introduce a un certo punto della narrazione (p. 140) un forte scarto temporale, che sorprende il lettore. Il cadavere di Salgari è stato ritrovato sulla collina, e la notizia è diventata di pubblico dominio. Improvvisamente la scena si trasferisce però all'interno di un'aula, durante una lezione scolastica. L'insegnante, conclusa l'ora dedicata alle operazioni, vorrebbe passare a spiegare la poesia del Carducci, ma tra gli scolari si sparge la voce della scomparsa del romanziere e i ragazzi - compiendo un atto veramente riprovevole per la morale del tempo - abbandonano l'aula ed escono per rendere omaggio al loro eroe (così faranno in una tavola a fianco, analogamente, degli operai che abbandoneranno la fabbrica). Non è difficile dedurre di quale classe si tratti: è la Terza della sezione Baretti di Torino, il maestro si chiama ovviamente Perboni. Siamo dunque precipitati indietro nel tempo, fra i protagonisti di Cuore (apparso nel 1886, ma le vicende narrate riguardano addirittura l'anno scolastico 1881-1882); i 
lettori facilmente riconoscono quegli scolari: il buon Garrone, il terribile Franti, lo studiosissimo Derossi, Bottini... Bacilieri non fa distinzione né di classe né di comportamenti, tant'è vero che Garrone e Franti (dunque in un certo senso i rappresentanti del bene e del male) decidono insieme di compiere un atto di indisciplina; anzi l'autore sceglie proprio questi scolari così diversi per censo e comportamento a rappresentare gli alunni d'Italia. Tutti, senza eccezioni, vogliono rendere onore al grande ispiratore dei loro sogni e delle loro avventure.

Anche in questa circostanza è evidente - almeno agli occhi non ingenui dello storico della letteratura - una sorta di contrapposizione fra il poeta-vate Giosuè Carducci (1835-1907), lo scrittore 'buonista' Edmondo De Amicis (1846-1908) e il popolarissimo Salgari. Gli scolari, gli operai, la gente normale insomma, tutti scelgono senza alcun dubbio lo scrittore veronese. Se è magari comprensibile l'accenno (in forma negativa) a un Carducci in fase calante di fronte alle novità letterarie che si profilavano all'orizzonte (in primis il Futurismo), ${ }^{6}$ è qui espresso, certo inconsciamente, un giudizio forse troppo severo su De Amicis. Ma si può certo scusare perché a Bacilieri premeva soprattutto rendere omaggio al suo eroe. Ormai morto e chiuso nella cassa, Salgari sembrava apparentemente un vinto, uno sconfitto, e invece restava nel cuore di tutti, restavano i suoi libri i suoi eroi. Questo è il messaggio finale, che in fondo non si può non condividere.

Ma non senza un'ultima postilla, che va oltre l'interpretazione di Bacilieri e tocca alcuni stereotipi tuttora presenti anche fra gli studiosi. De Amicis era morto a Bordighera l'11 marzo 1908; nel 1912, anno appunto della scomparsa di Salgari, Cuore aveva abbondantemente oltrepassato le 500 mila copie vendute e dunque non poteva non essere considerato un testo popolare sui generis. Era un prodotto editoriale altamente innovativo perché sapeva mescolare diverse tipologie (diario, lettere, racconti, e dal 1892 anche l'apparato iconografico) in nome di un'ideologia laica e patriottica funzionale all'educazione delle generazioni postunitarie. Ma quel fortunatissimo libro sarebbe diventato una sorta di prigione per il suo autore, tra l'altro spesso messo alla berlina dalla critica militante. De Amicis in effetti di lì a poco avrebbe prodotto un altro libro scolastico, il Romanzo di un maestro (Treves 1890) che per molti versi avrebbe potuto essere considerato un anti-Cuore, ma che passò quasi sotto silenzio; e nel frattempo aveva pubblicato un vero e proprio capolavoro, Sull'oceano (Treves 1889), un romanzo dedicato all'emigrazione italiana in Sudamerica in cui il sentimentalismo non aveva alcuno spazio. Poco dopo l'ex ufficiale dell'esercito (il "Capitan cortese" su cui ironizzava appunto il Carducci) avrebbe fatto il grande passo, avvicinandosi al socialismo e infine addirittura al pacifismo. Molta acqua era, ed è, dunque passata sotto i ponti, eppure De Amicis rimane inchiodato al solo Cuore.

Come Salgari, De Amicis viveva a Torino, e certo conosceva le opere del creatore di Sandokan, anche se i suoi figli apprezzavano soprattutto Jules Verne, con cui lo scrittore ebbe un memorabile incontro, raccontato in un capitolo delle sue Memorie. Nel capoluogo piemontese ebbe certamente modo di incrociare in qualche particolare occasione Salgari (ad esempio nello sferisterio per assistere a qualche incontro di pallone elastico), ${ }^{8}$ che a sua volta certamente conosceva alcuni libri di De Amicis, in particolare quelli di viaggio. ${ }^{9}$ I due forse non si incontrarono mai personalmente, ma non credo che Edmondo disprezzasse il più sfortunato collega, come è stato da qualcuno ipotizzato. ${ }^{10}$ 
18 Anche la biografia di De Amicis è molto distante dallo stereotipo dall'"Edmondo dei languori" ancora di conio carducciano ${ }^{11}$, e in alcuni aspetti ricorda l'esperienza di Salgari..$^{12}$ In essa andranno messe in conto la precoce perdita di un padre e un rapporto eccessivamente stretto (e difficile) con la madre. E un matrimonio coronato dalla nascita di due figli (Furio e Ugo), ma presto messo in crisi dalle scelte politiche di Edmondo e forse dal suo comportamento non sempre irreprensibile. E poi il momento più doloroso, il suicidio del ventiduenne figlio Furio (era il 15 novembre 1898), sparatosi su una panchina del parco del Valentino. Ciò accelerò la separazione dalla moglie, diventata quasi folle per il dolore ${ }^{13}$ e pronta ad accusare pubblicamente e senza tregua il marito, a sua volta sempre più chiuso nel suo dolore e progressivamente messo ai margini della scena letteraria. Insomma, una vita difficile, soprattutto negli ultimi anni. Un'esistenza senza particolari problemi economici, ma certo non da vincitore, rispetto al 'vinto' come Salgari. Una vita in gran parte ancora da scrivere e che meriterebbe di essere ripercorsa dalla mano intelligente di Bacilieri.

\section{NOTE}

1. G. De Luna, Quando la Storia è una buona Novel, in www.LaStampa.it Libri, 22 febbraio 2012. Verso la fine del suo intervento, De Luna propone altre interessanti riflessioni d'ordine generale su cui vale la pena di riflettere : "Importanti i contenuti di questi libri, ma altrettanto importante è il modello narrativo che propongono. Come ha scritto Paco Ignacio Taibo II nella prefazione al volume su Ciudad Juarez [Baudoin-Troubs, I sogni di Ciudad Juarez, Coconino-Fandango, 2012], questo linguaggio in cui si mescolano le riflessioni e i dialoghi in immagini, l'oggettività e la soggettività, potrebbe essere definito postrealista: laddove il realismo impone, ecco che il disegno suggerisce una definizione. Ha dentro più l'oralità che la scrittura, suggerisce più la visione che la lettura. Ed è per questo estremamente seduttivo ed efficace".

2. Cfr. S. Gonzato, La tempestosa vita di capitan Salgari, Vicenza, Neri Pozza, 2011, p. 198 e segg. Importante anche il libro di C. Gallo e G. Bonomi, Emilio Salgari. La macchina dei sogni, Milano, Rizzoli, 2011, con ricche annotazioni bibliografiche a cui senz'altro rinvio.

3. Tale operazione di inserimento di elementi in apparenza estranei al contesto si ripete per altro a p. 13 perché nella prima vignetta in altro compare, a sinistra, l'inconfondibile ritratto del torinese Guido Ceronetti, qui forse evocato come rappresentante della città, ma non solo : basti pensare ad un'opera noir come La vera storia di Rosa Vercesi e della sua amica Vittoria, Torino, Einaudi, 2000, ambientata nel capoluogo piemontese.

4. Come è noto, Salgari prima di morire lascerà una lettera in cui accuserà espressamente i suoi editori, rei d'essersi arricchiti “colla mia pelle"; il testo completo è in Gonzato, op. cit., p. 197, ma viene citato anche da Bacilieri a p. 137.

5. Tra essi sarà presente anche lo scrittore Salvatore Gotta (diventerà anch'egli, a suo modo, un'altra macchina da scrittura), che resterà fortemente colpito dal contrasto tra "l'educatore dei suoi sogni adolescenti", "baldo, audace, bello forte" come i suoi eroi il cadavere "nudo, sanguinolento, vecchio, miserabile come una bestia assassinata e abbandonata alle coltella dei sezionatori" (Gonzato, op. cit., p. 200). 
6. Ma per quest'ultimo, molto amato anche dai non letterati, si dovrà considerare (e spiegare) la straordinaria partecipazione popolare ai solenni funerali celebrati a Bologna il 18 febbraio 1907. Indispensabili su questo aspetto sono le riflessioni di Renato Serra,

7. E. De Amicis, Una visita a Jules Verne, in Id., Memorie, Milano, Treves, 1900, p. 237-257 (lo scritto è datato Ottobre 1895)

8. G. Spagarino Viglongo (Sulle orme di Salgari a Torino, "Ilcorsaronero", 6, marzo 2008, pp. 12-17 : 13: "Un altro degli abituali itinerari di Salgari ci conduce allo Sferisterio Vanchiglia di via Napione, lo stesso di cui era assiduo Edmondo De Amicis, per assistere al gioco del pallone elastico di cui entrambi erano patiti. Secondo la testimonianza del figlio, Salgari non ebbe mai modo - timore reverenziale? - di presentarsi a De Amicis - ch'era additato da tutti i torinesi - ma in realtà lo ammirava molto, anche se non era entusiasta del Cuore, 'troppo sentimentalume!' diceva. Qualche volta Salgari scommetteva ma infallibilmente perdeva e si consolava commentando che "non si può essere sempre fortunati nella vita"'.

9. Cfr. al riguardo B. Basile, De Amicis nei "Pirati della Malesia", "Filologia e critica", XXI,3, settembre-dicembre 1996, pp. 487-496 ; L. Tamburini, Salgari in Marocco sulle orme di De Amicis, "Studi Piemontesi", XXXI, fasc. 2, dicembre 2002, pp. 355-365. Tale dipendenza è confermata da M. Tropea,'I Predoni del Gran Deserto' (1911), vicenda di “vita eccentrica”, in Un po' prima della fine? Ultimi romanzi di Salgari tra novità e ripetizione (1908-1915), Atti del Convegno internazionale di Liège (18-19 febbraio 2009), a cura di Luciano Curreri e Fabrizio Foni, pp. 169-179 : 177-178.

10. E. Ferrero, Disegnare il vento, Torino, Einaudi, 2011, pp.110-111: "Il De Amicis arrivava allo sferisterio con un gruppetto di sodali che gli facevano corona e invece di interessarsi alla partita s'interessavano ai suoi commenti e imitavano il suo modo d'applaudire ed esclamare "oh che colpo! Che colpo maramaldo !". A fine partita poteva anche capitare che certi giocatori, ancora accaldati, andassero a salutarlo e a prendersi complimenti. Lui non osava avvicinarsi, tanto meno salutarlo. Figurarsi se il gran letterato si sarebbe degnato di scambiare qualche parola con lui, modesto badilante della penna". E cfr. invece quanto scrive S. Gonzato, La tempestosa vita di capitan Salgari, op. cit., 174 : "De Amicis e Salgari non si incontrarono mai. La cultura ufficiale ignorava lo scrittore veronese, oppure lo considerava una specie di fenomeno da baraccone". Forse entrambi dimenticano che Salgari non era proprio uno sconosciuto in campo letterario e che gli fu conferito il titolo di Cavaliere della Corona d'Italia : cfr. La regina e il capitano. Messaggi di Sua Maestà la Regina Margherita relativi a Emilio Salgari (1896-1900), a cura di Gian Paolo Marchi, Verona, Dipartimento di Romanistica, 2006.

11. Cfr. T. Barbieri, Carducci e De Amicis : documenti inediti, "Convivium”, XXVI, n.s., 5 settembreottobre 1958, pp.593-597, da integrare con la nota contenuta in Edmondo De Amicis. Mostra biobibliografica e iconografica a cura di Franco Contorbia, Città di Imperia, 1981, pp. 26-27.

12. Per essa rinvio agli studi di L. Tamburini, Mater dolorosa. Il calvario di Edmondo e Teresa De Amicis, "Studi Piemontesi", XVIII, 1, 1989, p. 25-47 ; Id., Teresa e Edmondo De Amicis. Dramma in un interno, Torino, Centro Studi Piemontesi, 1990 ; Id., Edmondo De Amicis. Metamorfosi di un borghese, Atripalda (Avellino), Mephite, 2008.

13. A. Brambilla, Edmondo (e Teresa) De Amicis "nel giardino della follia", in Id., De Amicis : paragrafi eterodossi, Modena, Mucchi, 1992, pp. 197-220. 


\section{AUTORI}

ALBERTO BRAMBILLA

Université de Franche-Comté 